\title{
Association of circulating resistin and adiponectin levels with Kawasaki disease: A meta-analysis
}

\author{
XINTIAN CAI, QING ZHU, TING WU, BIN ZHU, SHASHA LIU, SHANSHAN LIU, \\ XIAYIRE AIERKEN, AYGUZAL AHMAT and NANFANG LI \\ Center for Hypertension of The People's Hospital of Xinjiang Uygur Autonomous Region, \\ Hypertension Institute of Xinjiang, Urumqi, Xinjiang 830001, P.R. China
}

Received March 22, 2019; Accepted November 5, 2019

DOI: $10.3892 /$ etm.2019.8306

\begin{abstract}
The present meta-analysis was performed to examine the association between circulating blood adipokine levels and Kawasaki disease (KD). Studies were identified by searching various databases, including Web of Science, EMBASE, PubMed, Wanfang and China National Knowledge Infrastructure. After the studies were pooled, the mean difference (MD) and corresponding 95\% CI were calculated. Subgroup analyses and publication bias detection were also performed. The Cochrane Q test and $\mathrm{I}^{2}$ statistics were performed using Review Manager software (version 5.3) to test for heterogeneity. A Begg's test was used to assess publication bias and STATA software (version 12.0) was used for statistical analysis. The results revealed that the KD group exhibited higher levels of resistin compared with those in the healthy controls or disease controls (non-KD; $\mathrm{MD}=20.76$, 95\% CI=16.16-25.36, $\mathrm{P}<0.001 ; \mathrm{MD}=21.27,95 \% \mathrm{CI}=14.24-28.29$, $\mathrm{P}<0.001$, respectively). In addition, when compared with those in patients exhibiting non-coronary artery lesions (NCAL), those with coronary artery lesions (CAL) had higher levels of adiponectin and resistin $(\mathrm{MD}=1.00,95 \% \mathrm{CI}=0.06-1.96$, $\mathrm{P}=0.04 ; \mathrm{MD}=2.77,95 \% \mathrm{CI}=1.32-4.22, \mathrm{P}<0.001)$. Furthermore, compared with those in the inactive-phase group, patients in the active-phase group exhibited higher levels of resistin ( $\mathrm{MD}=17.73,95 \% \mathrm{CI}=12.82-22.65, \mathrm{P}<0.001)$. In conclusion, the present meta-analysis indicated that resistin levels were elevated in patients with KD. It was also revealed that circulating
\end{abstract}

Correspondence to: Professor Nanfang Li, Center for Hypertension of The People's Hospital of Xinjiang Uygur Autonomous Region, Hypertension Institute of Xinjiang, 91 Tianchi Road, Tianshan, Urumqi, Xinjiang 830001, P.R. China

E-mail: lnanfang2016@sina.com

Abbreviations: DC, disease control; HC, healthy control; $\mathrm{IL}$, interleukin; $\mathrm{KD}$, Kawasaki disease; MD, mean difference; NCAL, non-coronary artery lesions; RA, rheumatoid arthritis; SLE,systemic lupus erythematosus; TNF, tumor necrosis factor

Key words: resistin, adipokines, Kawasaki disease, meta-analysis resistin and adiponectin levels in the CAL group were significantly increased compared with those in patients with NCAL. Furthermore, the active group had higher levels of resistin than the inactive group. The results of these meta-analyses indicated that resistin may serve an important role in the pathogenesis of $\mathrm{KD}$ and may therefore be used as biomarkers for the diagnosis of KD, whereas adiponectin may only serve an important role in the pathogenesis of CAL and may therefore be used as a biomarker to distinguish CAL from NCAL.

\section{Introduction}

Kawasaki disease (KD) is a potentially fatal type of acute childhood vasculitis that primarily affects children under the age of 5 (1). KD frequently damages coronary arteries, resulting in a spectrum of injuries, including coronary artery aneurysms in severe cases (2). Although the pathogenesis of KD remains to be fully elucidated, the neutrophilic infiltration of arteries, acute/chronic vasculitis, as well as autoimmune and luminal myofibroblastic proliferation, are the major pathological features of KD (3).

Adipose tissue stores energy, regulates the endocrine system and is an important regulator of numerous important processes, including inflammation (4). Adipokines, derived from adipose tissues, are a class of soluble mediators that exert specific effects on immune responses. Certain adipokines, including resistin and adiponectin, serve as anti- and pro-inflammatory regulators. However, these adipokines represent only a small fraction of those identified and have recently been associated with inflammatory autoimmune diseases (5).

Human resistin is a $12.5-\mathrm{kDa}$ polypeptide that is produced primarily by bone marrow-derived monocytes with a degree of contribution from adipocytes $(6,7)$. It is widely accepted that resistin is involved in the inflammatory response (8). Resistin binds to human leukocyte Toll-like receptor 4 (TLR4), which regulates the production of various pro-inflammatory cytokines and interleukin (IL)-1 $\beta$, including IL-12 and IL-6, which in turn regulate the expression of resistin $(6,9)$.

The expression of adiponectin, a plasma protein of $30 \mathrm{kDa}$, decreases with obesity (10). Adiponectin has long been revealed as an anti-inflammatory adipokine, serving to protect blood vessel walls (11). It is used by endothelial cells to inhibit the expression of certain tumor necrosis factor (TNF)- $\alpha$-induced 
adhesion molecules, including vascular cell adhesion molecule-1, endothelial cell-leukocyte adhesion molecule-1 and intracellular adhesion molecule-1 (12). It may also lead to a reduction in the adhesion of monocytes to endothelial cells (13). Recent studies have indicated that adiponectin serves pro-inflammatory effects and that the serum concentrations of adiponectin are significantly elevated in patients with autoimmune diseases $(13,14)$. Adiponectin may therefore have a dual inflammatory function in autoimmune diseases, from inhibiting the expression of inflammatory cytokines and adhesion molecules to inducing the production of anti-inflammatory factors $(13,15)$.

Previous studies have investigated and compared the levels of circulating resistin and adiponectin in patients with KD and control subjects, with inconsistent results. These inconsistencies may be due to insufficient sample size leading to low statistical power and/or clinical heterogeneity (16-25). To overcome the limitations of previous research and address these inconsistencies, the present study performed a meta-analysis to provide a comprehensive overview. The levels of resistin and adiponectin in the serum of patients with KD were determined and compared with those of control subjects.

\section{Materials and methods}

Search strategy. To retrieve studies that met the inclusion criteria for the present meta-analysis, all reports assessing the association between serum resistin and adiponectin levels in patients with KD and controls [healthy control (HC) and disease control (DC, which was defined as those with clinical symptoms similar to KD but ultimately diagnosed as non-KD)], were reviewed. Literature searches were performed using Web of Science, EMBASE, PubMed, Wanfang and China National Knowledge Infrastructure databases (up to December 31, 2018). The following key words and subject terms were used in the search: 'Resistin', 'adiponectin', 'Kawasaki disease', 'mucocutaneous lymph node syndrome' and 'KD'. The search terms used were 'resistin' or 'adiponectin' crossed with 'Kawasaki disease' or 'mucocutaneous lymph node syndrome' or 'KD'.

The range of search strategies is broad and the desired information was potentially contained in any study evaluating serum adipokine levels in patients with KD, which was considered when selecting the key words for the search. The language was limited to English and Chinese. The bibliography of original research and review studies was also manually screened. If required, the authors of studies with insufficient data were contacted to request any missing data. The initial selection of studies was performed independently by XC and QZ. Disagreements between the reviewers were resolved by a third-party reviewer (NL) at a consensus meeting.

Inclusion criteria. The following inclusion criteria were utilized in the present study: i) Studies must be case-controlled, cross-sectional or cohort-based; ii) KD must have been diagnosed according to Japanese diagnostic criteria (26) or the 2017 American Heart Association common standard (27); iii) studies must report the mean \pm standard deviation (SD) of serum adipokine levels in patients with KD and control subjects; and iv) studies must describe the statistical methods and have performed the correct statistical analysis (28). When two or more studies reported the same data, the most recently updated study was considered. The two reviewers (XC and QZ) screened the titles and abstracts of the records retrieved from the literature. The full text of the potentially relevant studies was independently retrieved and evaluated by two reviewers (XC and QZ) and any discrepancies were resolved by third parties (NL).

Exclusion criteria. Studies were excluded from the present study if any of the following applied: i) Original studies containing no precise levels of serum resistin or adiponectin in patients with KD and control subjects; ii) no direct or indirect data for the mean \pm SD for continuous variables; iii) reviews or animal studies, reports with duplicate data or unpublished reports; and iv) a study design that was defective or of poor quality (29).

Data extraction. Two investigators anonymously separated information from all eligible studies selected according to the pre-defined criteria. The following data were extracted: Country or region of origin of the subjects, sex, mean age, body mass index $(B M I)$, serum resistin (mean $\pm \mathrm{SD}$ ), serum adiponectin levels (mean $\pm \mathrm{SD}$ ), name of the first author, year of publication, country, ethnicity, grouping and sample size. Researchers used spreadsheets to separate information independently from eligible studies. BMI, ethnicity and geographical location were considered during the data extraction process. If the standard error of the mean was reported, a statistical formula was used to calculate the SD. When the median, interquartile range or range was provided, the mean $\pm \mathrm{SD}$ was also calculated using the aforementioned formula (30,31). The Newcastle-Ottawa Quality Assessment Scale was used to assess the methodological quality of the studies selected (32).

Statistical analysis. All statistical analyses were performed using Review Manager software v.5.3 (Cochrane) and STATA v.12.0 software (Stata Corp. LP). A meta-analysis was performed to determine the possible association of resistin or adiponectin levels with KD. Results were expressed as the mean difference (MD) and 95\% CI. The Cochran Q and $\mathrm{I}^{2}$ statistics were used to assess the variation and heterogeneity within and between studies (33). The heterogeneity test was used to evaluate the null hypothesis, according to which all studies evaluated the same effect. When the Q-test result was $\mathrm{P}<0.05$ or $\mathrm{I}^{2}>50 \%$, it was indicated that heterogeneity existed. $\mathrm{I}^{2}$ values ranged from 0 to $100 \%$. In the present study, when $\mathrm{I}^{2}$ values were 25,50 and $75 \%$, the heterogeneity was referred to as low, medium and high estimates, respectively (34). In instances of heterogeneity between studies, a meta-analysis with a random-effects model was utilized to combine effectors. When no heterogeneity was identified, a fixed-effects model was used. Sensitivity analysis was performed to explore sources of heterogeneity. To assess publication bias, visual observations using the Egger's test and a funnel plot were used (35). $\mathrm{P}<0.05$ was considered to indicate a statistically significant difference.

\section{Results}

Studies included in the meta-analysis. A total of 86 studies were identified using electronic and manual retrieval methods, 22 of which were excluded as they reported the same data as other studies. Of the remaining 64 studies, 15 were excluded after reviewing the title and abstract and 39 were excluded due to 
Potential relevant articles identified from database search [Web of science, $n=24$; Embase, $n=20 ;$ Pubmed, $n=23$ : Wanfang, $n=8:$ CNKI, $n=11$ ] $(n=86)$

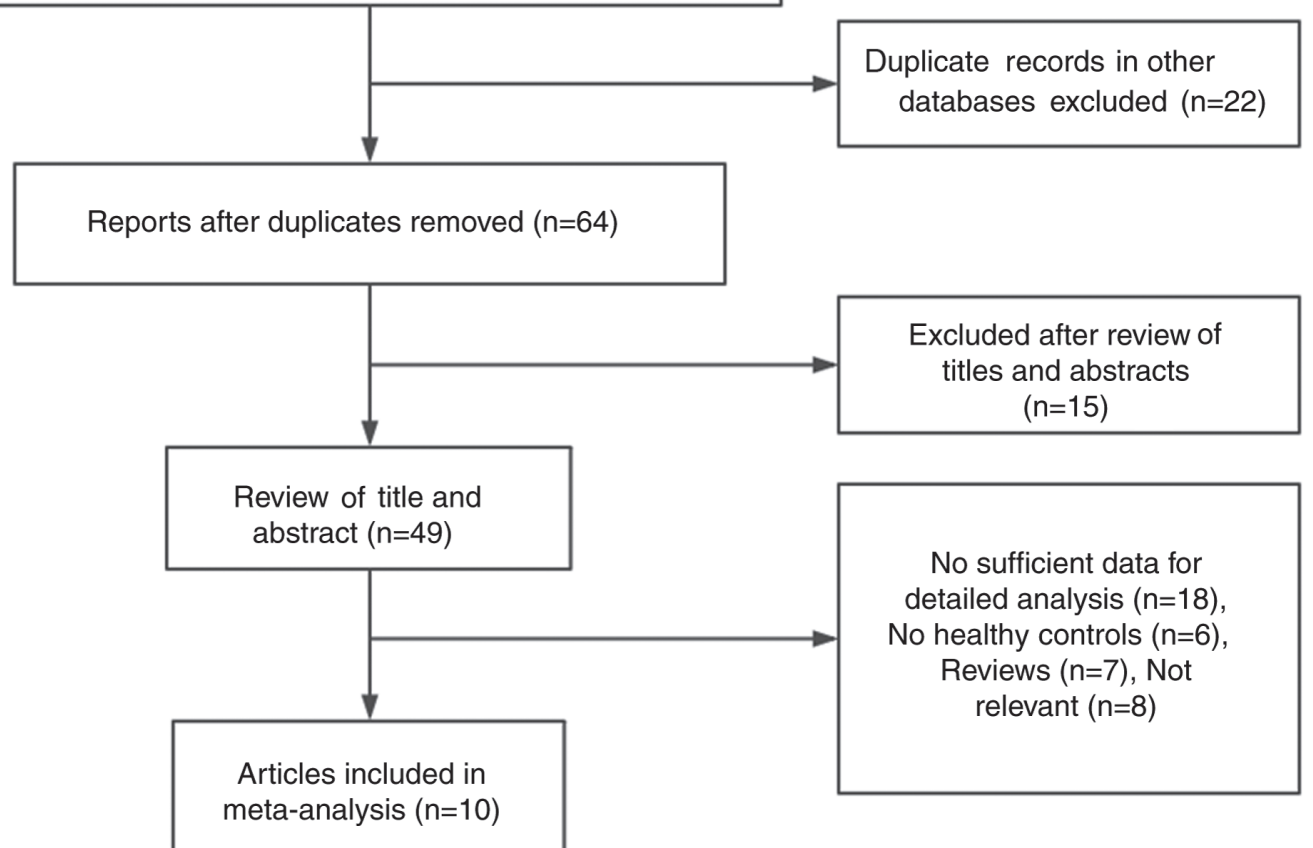

Figure 1. Flow chart of the study selection process. CNKI, Chinese National Knowledge Infrastructure.

repeated or irrelevant information, including animal or experimental studies, or were removed due to not being associated with resistin, adiponectin or KD. Finally, a total of 10 studiess met the inclusion criteria of the present meta-analysis (Table I). A flowchart diagram of the search process is presented in Fig. 1. Table I summarizes the basic features of the experimental and disease groups in the selected studies, as well as the specific types of adipokines assessed.

The levels of resistin (7 studies) (18-21,23-25) and adiponectin (6 studies) (16-18,20-22) were compared between KD groups and control groups. The quality evaluation scores of each study ranged from 6 to 9 and the methodological quality was high (Table I).

Meta-analysis of circulating adiponectin and resistin levels in $K D$ vs. HC groups. The meta-analysis revealed that the levels of resistin were significantly higher in the KD group compared with those in the HC group ( $\mathrm{MD}=20.76,95 \% \mathrm{CI}=16.16-25.36$, $\mathrm{P}<0.001$; Table II; Fig. 2A) (18-21,23-25). However, no statistically significant differences in adiponectin levels were identified between the KD group and the $\mathrm{HC}$ group ( $\mathrm{MD}=-3.03$, 95\% CI=-6.46-0.40, $\mathrm{P}=0.08$; Table II) $(16-18,20-22)$.

Meta-analysis of circulating adiponectin and resistin levels in $K D$ group vs. DC group. The present meta-analysis revealed that the levels of resistin were significantly higher in the KD group compared with those in the $\mathrm{DC}$ group $(\mathrm{MD}=21.27$, 95\% CI=14.24-28.29, P<0.001; Table II; Fig. 2B) $(18,19,21)$. However, no statistically significant differences in adiponectin levels were identified between the KD group and the DC group $(\mathrm{MD}=-5.49,95 \% \mathrm{CI}=-11.28-0.31, \mathrm{P}=0.06$; Table II) $(16-18,21)$.
Meta-analysis of circulating adiponectin and resistin levels in CAL group vs. NCAL group. The present meta-analysis revealed that the levels of adiponectin and resistin were significantly associated with CAL in patients with $\mathrm{KD}(\mathrm{MD}=1.00$, 95\% $\mathrm{CI}=0.06-1.94, \mathrm{P}=0.04 ; \mathrm{MD}=2.77,95 \% \mathrm{CI}=1.32-4.22$, $\mathrm{P}<0.001$; Table II; Fig. 2C and D) (17,20-22,24,25).

Meta-analysis of circulating adiponectin and resistin levels in the active group vs. inactive group. The results of the meta-analysis revealed that the levels of resistin were significantly higher in the active group (within 11 days of onset) when compared with those in the inactive group (after 12 days of onset; $M D=17.73$, 95\% CI=12.82-22.65, P<0.001; Table II; Fig. 2E) $(18,19,23,24)$. However, adiponectin levels were not significantly different between patients with active or inactive $\mathrm{KD}(\mathrm{MD}=-1.54$, 95\% CI=-3.08-0.00, $\mathrm{P}=0.05$; Table II) $(16,18,23)$.

Sensitivity analyses and publication bias. To evaluate the stability of the results of the meta-analysis, sensitivity analyses were performed on resistin and adiponectin data. The results revealed that the overall statistical significance did not change when any single study was omitted (Fig. 3A and B). Therefore, the results of the present meta-analysis were deemed to be relatively reliable and credible. To assess publication bias, a Begg's test was performed, which included at least three studies. It was determined that the distribution of the funnel plot was slightly asymmetrical (Figs. S1-S6). Therefore, in the following subgroup analyses, a Begg's test was used to evaluate the asymmetry of the funnel plot. The results of the Begg's test for publication bias revealed that there was almost no publication bias in the present analysis (Table II). 


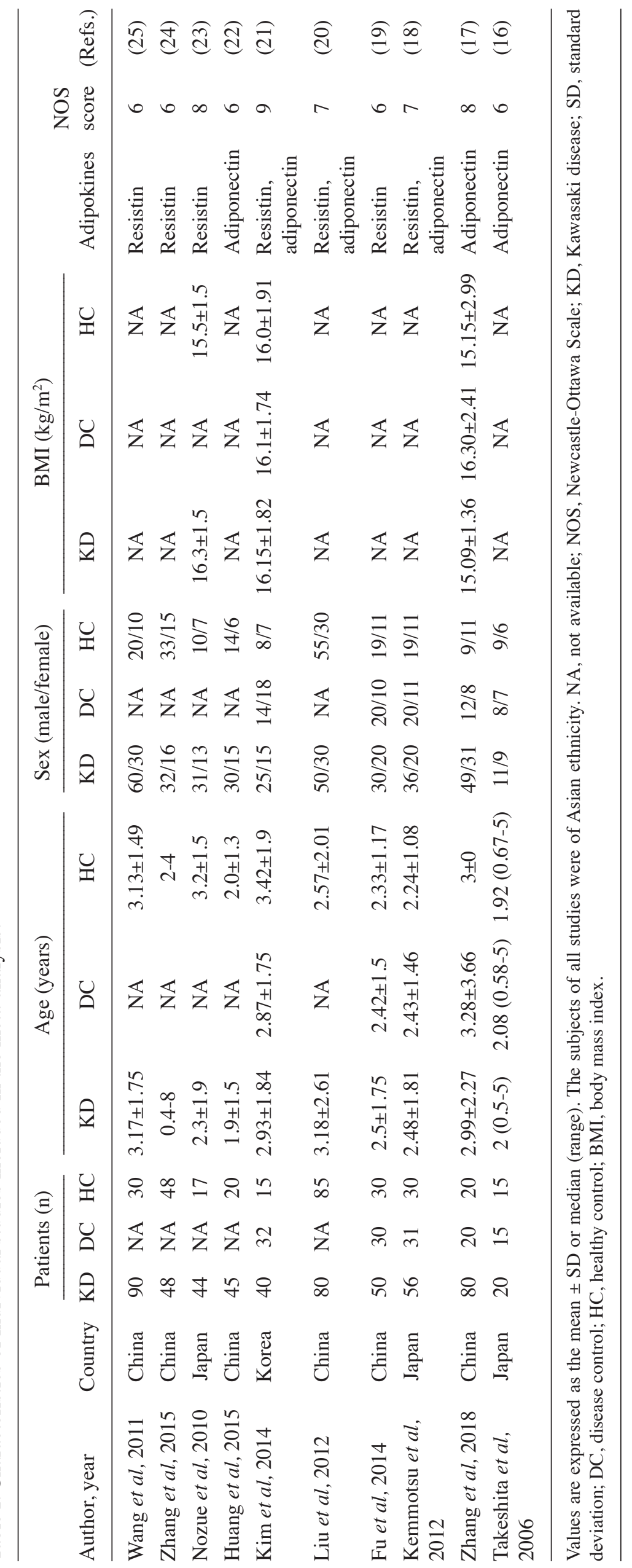


Table II. Subgroup analysis of serum adipokine levels in patients with KD and control subjects.

A, Adipokines in KD vs. controls

\begin{tabular}{|c|c|c|c|c|c|c|c|c|}
\hline \multirow[b]{2}{*}{ Comparison } & \multirow{2}{*}{$\begin{array}{l}\text { Studies } \\
\text { (n) }\end{array}$} & \multirow{2}{*}{$\begin{array}{l}\text { Patients } \\
\text { (n) }\end{array}$} & \multicolumn{2}{|c|}{ Test of association } & \multicolumn{3}{|c|}{ Test of heterogeneity } & \multirow{2}{*}{$\begin{array}{c}\text { Begg's test } \\
\text { P-value }\end{array}$} \\
\hline & & & $\mathrm{MD}(95 \% \mathrm{CI})$ & P-value & Model & $\mathrm{I}^{2}(\%)$ & $\mathrm{P}$-value & \\
\hline \multicolumn{9}{|l|}{ Resistin } \\
\hline KD vs. HC & 7 & $408 / 255$ & $20.76(16.16,25.36)$ & $<0.001$ & Random & 94 & $<0.001$ & 0.133 \\
\hline KD vs. DC & 3 & $146 / 93$ & $21.27(14.24,28.29)$ & $<0.001$ & Random & 59 & 0.09 & 0.296 \\
\hline \multicolumn{9}{|l|}{ Adiponectin } \\
\hline KD vs. HC & 6 & $321 / 185$ & $-3.03(-6.46,0.40)$ & 0.08 & Random & 95 & $<0.001$ & \\
\hline KD vs. DC & 4 & $196 / 98$ & $-5.49(-11.28,0.31)$ & 0.06 & Random & 92 & $<0.001$ & \\
\hline
\end{tabular}

B, Subgroup comparisons

\begin{tabular}{|c|c|c|c|c|c|c|c|c|}
\hline \multirow[b]{2}{*}{ Comparison } & \multirow{2}{*}{$\begin{array}{c}\text { Studies } \\
\text { (n) }\end{array}$} & \multirow{2}{*}{$\begin{array}{l}\text { Patients } \\
\text { (n) }\end{array}$} & \multicolumn{2}{|c|}{ Test of association } & \multicolumn{3}{|c|}{ Test of heterogeneity } & \multirow{2}{*}{$\begin{array}{c}\text { Begg's test } \\
\text { P-value }\end{array}$} \\
\hline & & & $\mathrm{MD}(95 \% \mathrm{CI})$ & P-value & Model & $\mathrm{I}^{2}(\%)$ & P-value & \\
\hline \multicolumn{9}{|c|}{ CAL vs. NACL } \\
\hline Adiponectin & 4 & $109 / 136$ & $1.00(0.06,1.94)$ & 0.04 & Fixed & 13 & 0.33 & 0.308 \\
\hline Resistin & 4 & $117 / 141$ & $2.77(1.32,4.22)$ & $<0.001$ & Fixed & 8 & 0.35 & 0.734 \\
\hline \multicolumn{9}{|c|}{ Active vs. inactive group } \\
\hline Adiponectin & 3 & $121 / 121$ & $-1.54(-3.08,0.00)$ & 0.05 & Random & 73 & 0.03 & 0.602 \\
\hline Resistin & 4 & $186 / 186$ & $17.73(12.82,22.65)$ & $<0.001$ & Random & 83 & $<0.001$ & 0.308 \\
\hline
\end{tabular}

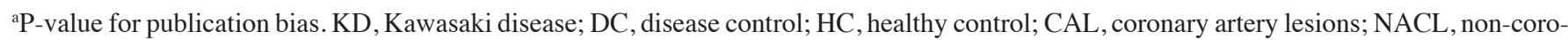
nary artery lesions; MD, mean difference; Fixed, fixed-effects model; Random, random-effects model.

\section{Discussion}

For numerous years, adipose tissue has been considered to be a bodily energy store, also providing mechanical defense against damage and serving as a temperature regulator (36). With the discovery of adipokines, an increasing number of studies have revealed that white adipose tissue is an active endocrine organ (37). It secretes a variety of adipokines, including ubiquitous molecules, e.g. TNF- $\alpha$ and IL-6, as well as specific molecules, e.g. adiponectin (38). Based on their effects on inflammation, these adipokines may be divided into two types: One that exerts pro-inflammatory effects only (including resistin) and another that exerts anti-inflammatory and pro-inflammatory effects (including adiponectin) (12,37,39). The expression and pathogenic effects of adipokines, including resistin and adiponectin, have been evaluated in a variety of autoimmune rheumatic diseases, particularly in KD (40).

Recent studies have demonstrated that resistin binds to TLR4, which leads to the production of pro-inflammatory cytokines by various types of human leukocyte, including IL-12, IL-6 and IL-1 $\beta(5,14,41)$. These cytokines, in turn, further potentiate the expression of resistin. Adipocytes treated with resistin produce pro-inflammatory responses (release of TNF- $\alpha$ and IL-6), peripheral blood mononuclear cells (TNF- $\alpha$, IL- 6 and IL-1 $\beta$ ) and hepatic stellate cells (IL-8 and monocyte chemoattractant protein 1) in vitro $(42,43)$. These effects on cytokine secretion occur via traditional NF- $\mathrm{B}$-mediated signaling (43). In addition, increased levels of circulating resistin are associated with markers of inflammation and joint destruction in rheumatoid arthritis (RA). Furthermore, intra-articular injections of resistin have been demonstrated to induce arthritis. By contrast, anti-TNF- $\alpha$ treatment significantly reduced the expression of resistin in RA $(38,44,45)$. Although reports of resistin expression in systemic lupus erythematosus (SLE) are not consistent, it has been suggested that the purpose of increased resistin levels may go beyond those of a biomarker to include a functional role in the progression of SLE $(40,46)$. Patients with ankylosing spondylitis and psoriasis also exhibit higher levels of resistin and serum resistin levels are also closely associated with Sjögren's syndrome and inflammatory bowel disease (47-51). These data provide strong evidence that resistin may be a useful marker for the general inflammatory state in autoimmunity and that its expression may be decreased after treatment or remission. This is in agreement with the conclusions of the present meta-analysis. Resistin may not only identify patients with KD, those with common fever and healthy controls, but may also differentiate between active and inactive $\mathrm{KD}$, and determine coronary artery involvement in patients with KD. Furthermore, resistin may serve an important role in the autoimmune inflammation that mediates KD.

Previous studies have revealed that adiponectin produces an anti-inflammatory effect (52-54). Adiponectin is also an 


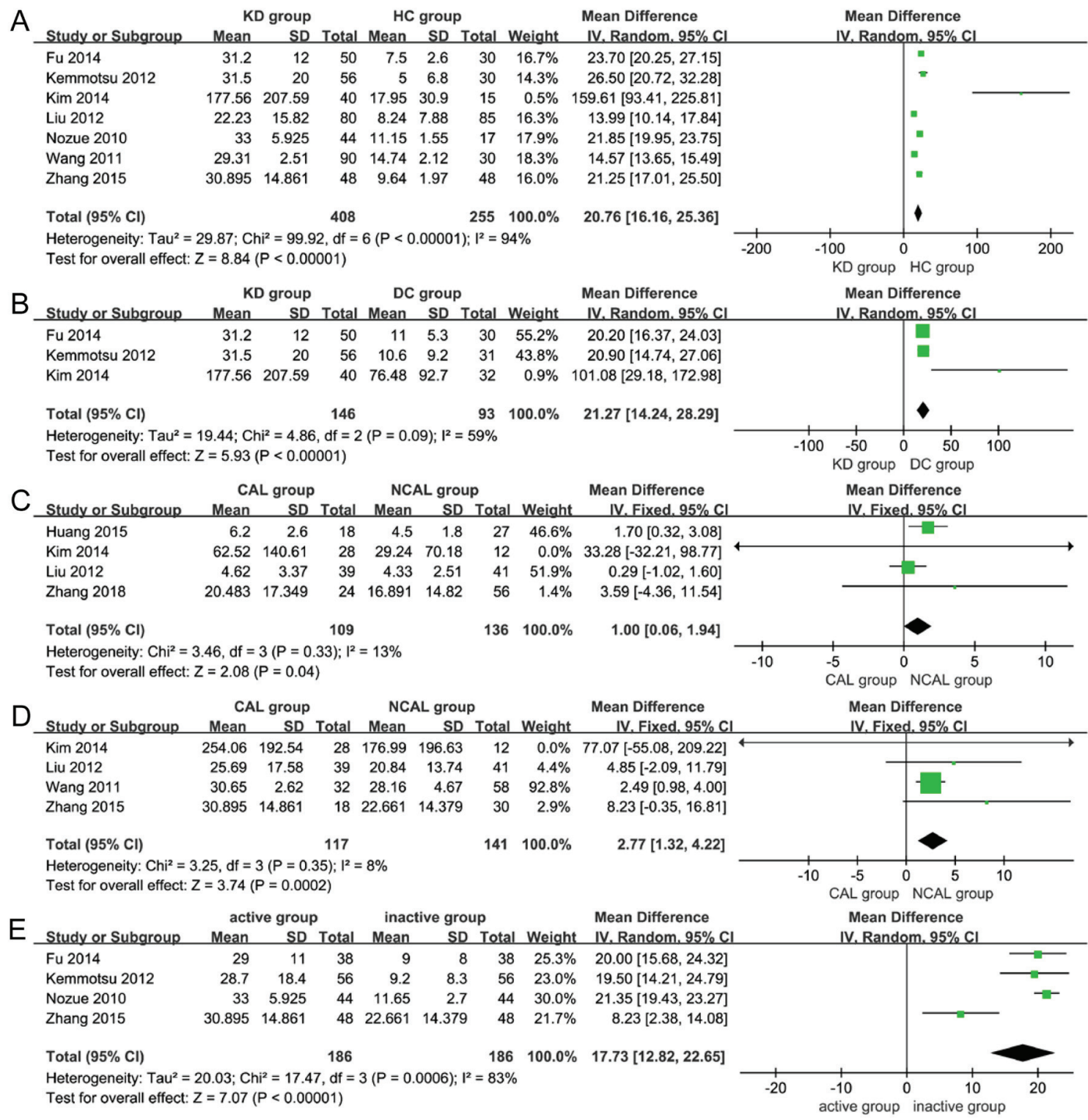

Figure 2. Subgroup analyses for the association of serum adiponectin or resistin levels in patients with KD. Meta-analysis of (A) circulating resistin levels in KD group vs. HC group; (B) circulating resistin levels in KD group vs. DC group; (C) circulating adiponectin levels in CAL group vs. NCAL group; (D) circulating resistin levels in CAL group vs. NCAL group; and (E) circulating resistin levels in active vs. inactive disease group. Fixed, fixed-effects model; Random, random-effects model; KD, Kawasaki disease; NCAL, non-coronary artery lesions; HC, healthy control; DC, disease control; IV, inverse variance; $\mathrm{SD}$, standard deviation; DF, degrees of freedom; CAL, coronary artery lesions.

insulin-sensitized adipokine (53). Higher adiponectin levels enhance insulin sensitivity, while lower adiponectin plasma levels are associated with type 2 diabetes, dyslipidemia, hypertension and coronary artery disease (55). In addition, studies have indicated that adiponectin inhibits the expression of TNF- $\alpha$ and regulates the conversion of macrophages to foam cells, thereby protecting the blood vessel wall (53-55). It has also been determined that adiponectin exerts an anti-inflammatory effect on the immune system, inhibiting the differentiation and activation of M1 macrophages by lowering inflammatory cytokine levels in macrophages, including TNF- $\alpha$ and IL-6, while promoting the proliferation of M2 macrophages and the secretion of certain anti-inflammatory markers, including IL-10 (55). TNF- $\alpha$ and IL-6 also inhibit the production of adiponectin, indicating that negative feedback regulation exists between adiponectin and inflammatory cytokines (56).
Recently, adiponectin has been demonstrated to activate the major signaling pathways that inhibit the proliferation of cancer cells and induce apoptosis of inflammatory cells (57). In addition, other studies have confirmed the pro-inflammatory effect of adiponectin $(58,59)$. Although adiponectin serves a protective role in certain metabolic disorders, including obesity and insulin resistance, a growing number of studies have indicated that serum or local tissue adiponectin levels in patients with autoimmune or chronic inflammatory diseases are significantly elevated $(52,60,61)$. Examples are RA, SLE and inflammatory bowel disease, all of which do not appear to be significantly correlated with obesity. The debate over whether adiponectin levels are elevated or decreased in autoimmune diseases continues in patients with $\operatorname{KD}(52,60,61)$. Huang et al $(22)$ and Kemmotsu et al (18) suggested that the levels of adiponectin in the circulating blood of patients with KD were higher compared 
A

Meta-analysis estimates, respective study is omitted

I Lower Cl limit o Estimate

I Upper Cl limit

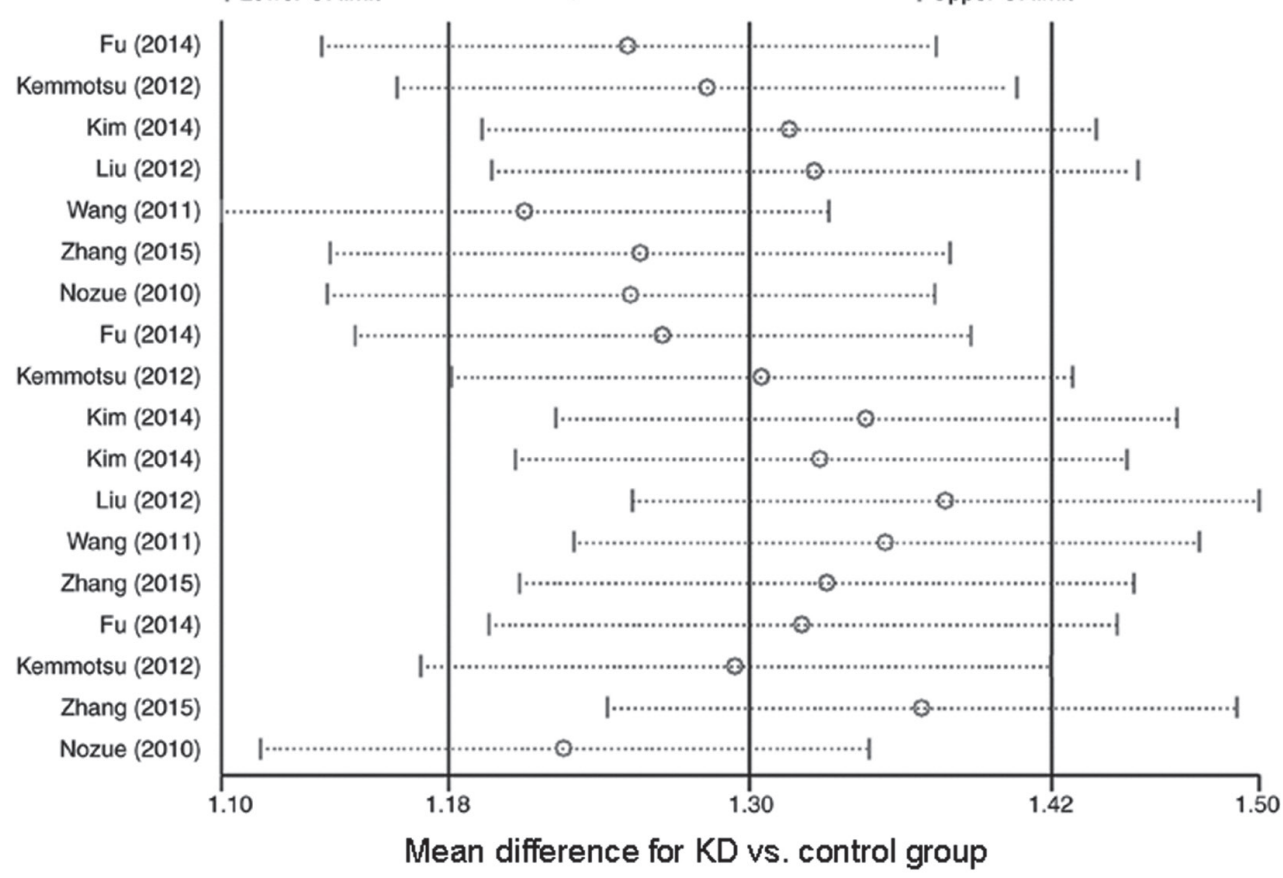

B Meta-analysis estimates, respective study is omitted

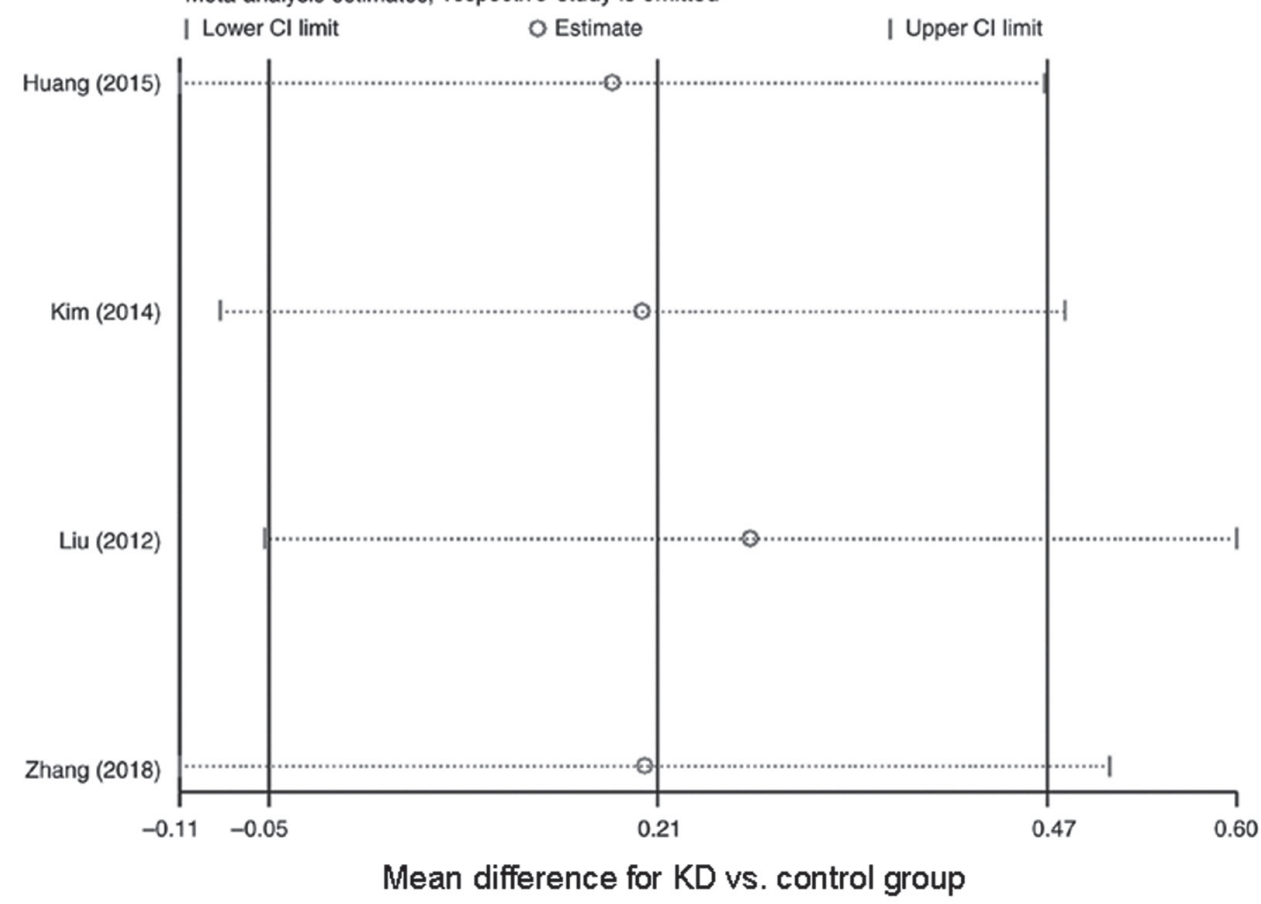

Figure 3. Sensitivity analyses were performed with one study excluded at a time. (A) Sensitivity analyses for circulating resistin levels (mean difference for KD vs. control group); and (B) sensitivity analyses for circulating adiponectin levels (mean difference for KD vs. control group). KD, Kawasaki disease.

with those in the control group. However, Takeshita et al (16) demonstrated that circulating levels of adiponectin in patients with KD were lower compared with those in the control group. The results of the present meta-analysis did not indicate that adiponectin levels were elevated or decreased in patients with $\mathrm{KD}$, which is a subject that requires exploration in future studies. However, the present meta-analysis of patients with KD and CAL revealed that patients with CAL frequently exhibited a significant increase in adiponectin levels, suggesting that adiponectin levels may be used to predict the presence of CAL in patients with KD. The differences between the adiponectin levels in the acute and inactive group were bordering on being significant, this may have been due to the small sample size and different detection methods; thus, this should be investigated further in the future.

However, the present meta-analysis has certain limitations. Heterogeneity of data/between studies may exist, which may affect the conclusions of the meta-analysis performed. However, 
random-effects models were utilized to address this limitation. In addition, any literature-based review and meta-analysis may have reporter bias (occurring due to only including studies published in English and Chinese). Furthermore, in most of the studies included in the present meta-analysis, there was no mention of gender differences between resistin and adiponectin levels detected. Finally, the sample size of certain selected studies was relatively small. These limitations may affect the final conclusions of the present study. However, the present meta-analysis also has advantages. To the best of our knowledge, the present meta-analysis was the first to comprehensively compile evidence on the adipokine status of patients with KD. Similarly, compared with individual studies, the results of multiple independent analyses improved the statistical power and resolution of data, resulting in higher accuracy. However, due to the aforementioned limitations, further research is required to address any discrepancies and opportunities bias. In addition, research on the underlying mechanisms of the effects of multiple adipokines in patients with KD is also required (62-65).

In conclusion, the present meta-analysis revealed an association of serum resistin and adiponectin levels with KD. The results also demonstrated that serum resistin levels in patients with KD were significantly higher compared with those in HCs and DCs. However, no significant differences in adiponectin levels were identified in patients with KD vs. controls. Furthermore, in patients with $\mathrm{KD}$, the resistin levels in the CAL group and active disease group were higher compared with those in the NCAL group and inactive stage group, respectively. Adiponectin levels were also higher in the CAL group compared with those in the NCAL group. The results of the present meta-analysis indicated that resistin may have an immunological effect in the pathogenesis of KD, which in turn suggests that serum resistin may be a potential marker for KD and serve as an effective therapeutic target. Adiponectin may be closely associated with the involvement of CAL in patients with KD. However, further studies are required to determine whether resistin or adiponectin directly contributes to the pathogenesis of KD.

\section{Acknowledgements}

Not applicable.

\section{Funding}

The present study was supported by The Xinjiang Uygur Autonomous Region Natural Science Fund (grant no. 2018D01C117).

\section{Availability of data and materials}

All data generated and/or analyzed during this study are included in this published study.

\section{Authors' contributions}

$\mathrm{XC}$ and NL were involved in data management, statistics, the first and final drafts, and approval. QZ and TW verifed the data extracted following the literature search, monitored the study and were involved in the final draft. BZ, ShasL, ShanL, XA and AA contributed to designing the search strategy. BZ and ShasL conducted the searches and aided with the statistical analysis. XA, ShanL and AA performed the data extraction and contributed to quality assessment. All authors contributed to drafting and revising the manuscript.

\section{Ethics approval and consent to participate}

Not applicable.

\section{Patient consent for publication}

Not applicable.

\section{Competing interests}

The authors declare that they have no competing interests.

\section{References}

1. Dietz SM, Tacke CE,Hutten BA and Kuijpers TW: Peripheral endothelial (Dys)function, arterial stiffness and carotid intima-media thickness in patients after kawasaki disease: A systematic review and meta-analyses. PLoS One 10: e0130913, 2015.

2. Chen KY, Curtis N, Dahdah N, Kowalski R, Cheung M and Burgner DP: Kawasaki disease and cardiovascular risk: A comprehensive review of subclinical vascular changes in the longer term. Acta Paediatr 105: 752-761, 2016.

3. Zhu FH and Ang JY: The clinical diagnosis and management of kawasaki disease: A review and update. Curr Infect Dis Rep 18: 32, 2016.

4. Chung HS and Choi KM: Adipokines and myokines: A pivotal role in metabolic and cardiovascular disorders. Curr Med Chem 25: 2401-2415, 2018.

5. Hutcheson J: Adipokines influence the inflammatory balance in autoimmunity. Cytokine 75: 272-279, 2015.

6. Jamaluddin MS, Weakley SM, Yao Q and Chen C: Resistin: Functional roles and therapeutic considerations for cardiovascular disease. Br J Pharmacol 165: 622-632, 2012.

7. Degawa-Yamauchi M, Bovenkerk JE, Juliar BE, Watson W, Kerr K, Jones R, Zhu Q and Considine RV: Serum resistin (FIZZ3) protein is increased in obese humans. J Clin Endocrinol Metab 88: 5452-5455, 2003.

8. Bokarewa M, Nagaev I, Dahlberg L, Smith U and Tarkowski A: Resistin, an adipokine with potent proinflammatory properties. J Immunol 174: 5789-5795, 2005.

9. Tarkowski A, Bjersing J, Shestakov A and Bokarewa MI: Resistin competes with lipopolysaccharide for binding to toll-like receptor 4 . J Cell Mol Med 14: 1419-1431, 2010.

10. Sun Y, Xun K, Wang C, Zhao H, Bi H, Chen X and Wang Y: Adiponectin, an unlocking adipocytokine. Cardiovasc Ther 27: 59-75, 2009.

11. Scotece M, Conde J, López V, Lago F, Pino J, Gómez-Reino JJ and Gualillo O: Adiponectin and leptin: New targets in inflammation. Basic Clin Pharmacol Toxicol 114: 97-102, 2014.

12. Francisco V, Ruiz-Fernández C, Pino J, Mera A, González-Gay MA, Gómez R, Lago F, Mobasheri A and Gualillo O: Adipokines: Linking metabolic syndrome, the immune system, and arthritic diseases. Biochem Pharmacol 165: 196-206, 2019.

13. Frommer KW, Zimmermann B, Meier FM, Schröder D, Heil M, Schäffler A, Büchler C, Steinmeyer J, Brentano F, Gay S, et al: Adiponectin-mediated changes in effector cells involved in the pathophysiology of rheumatoid arthritis. Arthritis Rheumatol 62: 2886-2899, 2010

14. Versini M, Jeandel PY, Rosenthal E and Shoenfeld Y: Obesity in autoimmune diseases: Not a passive bystander. Autoimmun Rev 13: 981-1000, 2014.

15. Stofkova A: Leptin and adiponectin: From energy and metabolic dysbalance to inflammation and autoimmunity. Endocr Regul 43: 157-168, 2009.

16. Takeshita S, Takabayashi $\mathrm{H}$ and Yoshida N: Circulating adiponectin levels in Kawasaki disease. Acta Paediatr 95: 1312-1314, 2006. 
17. Zhang XY, Yang TT, Hu XF, Wen Y, Fang F and Lu HL: Circulating adipokines are associated with Kawasaki disease. Pediatr Rheumatol Online J 16: 33, 2018.

18. Kemmotsu Y, Saji T, Kusunoki N, Tanaka N, Nishimura C, Ishiguro A and Kawai S: Serum adipokine profiles in Kawasaki disease. Mod Rheumatol 22: 66-72, 2012.

19. Fu SF, Yu DL, Lu DY and Chen FY: Changes in serum levels of resistin and visfatin in pediatric patients with acute Kawasaki disease following intravenous immune globulin treatment. Zhongguo Dang Dai Er Ke Za Zhi 16: 44-47, 2014 (In Chinese).

20. Liu R, He B, Gao F, Liu Q and Yi Q: Relationship between adipokines and coronary artery aneurysm in children with Kawasaki disease. Transl Res 160: 131-136, 2012.

21. Kim HJ, Choi EH and Kil HR: Association between adipokines and coronary artery lesions in Children with Kawasaki Disease. J Korean Med Sci 29: 1385-1390, 2014.

22. Huang M, Dong QH, Jiang HY, Zhang JY and Shi XJ: Serum adiponectin levels in children with Kawasaki disease. Zhongguo Dang Dai Er Ke Za Zhi 17: 35-39, 2015 (In Chinese).

23. Nozue H, Imai H, Saitoh H, Aoki T, Ichikawa K and Kamoda T: Serum resistin concentrations in children with Kawasaki disease. Inflamm Res 59: 915-920, 2010.

24. Zhang LR, Qiu WG, Pan XF and Liu RY: Changes of serum resistin hemoglobin level in children with kawasaki disease and its relationship with the degree of coronary artery dilation. Shandong Yi Yao Za Zhi 55: 89-91, 2015 (In Chinese).

25. Wang XY, Huang YM and Zhang WL: Changes of contents of endothelin and resistin in children with kawasaki disease. Zhonghua Lin Chuang Yi Shi Za Zhi (Dian Zi Ban) 05: 170-171, 2011 (In Chinese).

26. JCS Joint Working Group: Guidelines for diagnosis and management of cardiovascular sequelae in Kawasaki disease (JCS 2013). Digest version. Circ J 78: 2521-2562, 2014.

27. Sosa T, Brower L and Divanovic A: Diagnosis and management of Kawasaki disease. JAMA Pediatr 173: 278-279, 2019.

28. Jiang S, Song K, Feng S and Shi YB: Association between serum leptin levels and peritoneal dialysis: A meta-analysis. Exp Ther Med 10: 300, 2015 .

29. Li D, Qian W, Li RR, Zhang J, Li KE and Wu Y: Correlation between lung neoplasm and serum level of osteopontin: A meta-analysis. Biomed Rep 4: 567-572, 2016.

30. Ridout KK, Ridout SJ, Price LH, Sen S and Tyrka AR: Depression and telomere length: A meta-analysis. J Affect Disord 191: 237-247, 2016.

31. Hozo SP, Djulbegovic B and Hozo I: Estimating the mean and variance from the median, range, and the size of a sample. BMC Med Res Methodol 5: 13, 2005.

32. Stang A: Critical evaluation of the Newcastle-Ottawa scale for the assessment of the quality of nonrandomized studies in meta-analyses. Eur J Epidemiol 25: 603-605, 2010.

33. Zhu B, Zhu Q, Li N, Wu T, Liu S and Liu S: Association of serum/plasma high mobility group box 1 with autoimmune diseases: A systematic review and meta-analysis. Medicine (Baltimore) 97: e11531, 2018.

34. Lee YH and Song GG: Urinary MCP-1 as a biomarker for lupus nephritis: A meta-analysis. Z R heumatol 76: 357-363, 2017.

35. Song F and Gilbody S: Bias in meta-analysis detected by a simple, graphical test. Increase in studies of publication bias coincided with increasing use of meta-analysis. BMJ 316: 471, 1998.

36. Greenberg AS and Obin MS: Obesity and the role of adipose tissue in inflammation and metabolism. Am J Clin Nutr 83 (Suppl): S461-S465, 2006.

37. Kajimura S: Adipose tissue in 2016: Advances in the understanding of adipose tissue biology. Nat Rev Endocrinol 13: 69-70, 2017.

38. Gremese E, Tolusso B, Gigante MR and Ferraccioli G: Obesity as a risk and severity factor in rheumatic diseases (autoimmune chronic inflammatory diseases). Front Immunol 5: 576, 2014.

39. Huang Q, Tao SS, Zhang YJ, Zhang C, Li LJ, Zhao W, Zhao MQ, Li P, Pan HF, Mao C and Ye DQ: Serum resistin levels in patients with rheumatoid arthritis and systemic lupus erythematosus: A meta-analysis. Clin Rheumatol 34: 1713-1720, 2015.

40. Li HM, Zhang TP, Leng RX, Li XP, Li XM, Liu HR, Ye DQ and Pan HF: Emerging role of adipokines in systemic lupus erythematosus. Immunol Res 64: 820-830, 2016.

41. Sawicka K and Krasowska D: Adipokines in connective tissue diseases. Clin Exp Rheumatol 34: 1101-1112, 2016.

42. Guerrero-García JDJ, Carrera-Quintanar L, López-Roa RI, Márquez-Aguirre AL and Ortuño-Sahagún D: Multiple sclerosis and obesity: Possible roles of adipokines. Mediators Inflamm 2016: 4036232, 2016.
43. Guzik TJ, Skiba DS, Touyz RM and Harrison DG: The role of infiltrating immune cells in dysfunctional adipose tissue. Cardiovasc Res 113: 1009-1023, 2017.

44. Sato H, Muraoka S, Kusunoki N, Masuoka S, Yamada S, Ogasawara H, Imai T, Akasaka Y, Tochigi N, Takahashi H, et al: Resistin upregulates chemokine production by fibroblast-like synoviocytes from patients with rheumatoid arthritis. Arthritis Res Ther 19: 263, 2017.

45. Senolt L, Housa D, Vernerová Z, Jirásek T, Svobodová R, Veigl D, Anderlová K, Müller-Ladner U, Pavelka K and Haluzík M: Resistin in rheumatoid arthritis synovial tissue, synovial fluid and serum. Ann Rheum Dis 66: 458, 2007.

46. Hutcheson J, Ye Y, Han J, Arriens C, Saxena R, Li QZ, Mohan C and $\mathrm{Wu} \mathrm{T}$ : Resistin as a potential marker of renal disease in lupus nephritis. Clin Exp Immunol 179: 435-443, 2015.

47. Syrbe U, Callhoff J, Conrad K, Poddubnyy D, Haibel H, Junker S, Frommer KW, Müller-Ladner U, Neumann E and Sieper J: Serum adipokine levels in patients with ankylosing spondylitis and their relationship to clinical parameters and radiographic spinal progression. Arthritis Rheumatol 67: 678-685, 2015.

48. Karmiris $\mathrm{K}$ and Koutroubakis IE: Resistin: Another rising biomarker in inflammatory bowel disease. Eur J Gastroenterol Hepatol 19: 1035-1037, 2007.

49. Konrad A, Lehrke M, Schachinger V, Seibold F, Stark R, Ochsenkühn T, Parhofer KG, Göke B and Broedl UC: Resistin is an inflammatory marker of inflammatory bowel disease in humans. Eur J Gastroenterol Hepatol 19: 1070-1074, 2007.

50. Boström EA, D'Elia HF, Dahlgren U, Simark-Mattsson C, Hasséus B, Carlsten H, Tarkowski A and Bokarewa M: Salivary resistin reflects local inflammation in Sjögren's syndrome. J Rheumatol 35: 2005-2011, 2008.

51. Huang H, Shen E, Tang S, Tan X, Guo X, Wang Q and Ding H: Increased serum resistin levels correlate with psoriasis: A meta-analysis. Lipids Health Dis 14: 44, 2015.

52. Peng YJ, Shen TL, Chen YS, Mersmann HJ, Liu BH and Ding ST: Adiponectin and adiponectin receptor 1 overexpression enhance inflammatory bowel disease. J Biomed Sci 25: 24, 2018.

53. Luo Y and Liu M: Adiponectin: A versatile player of innate immunity. J Mol Cell Biol 8: 120-128, 2016.

54. Okamoto Y, Kihara S, Funahashi T, Matsuzawa Y and Libby P: Adiponectin: A key adipocytokine in metabolic syndrome. Clin Sci (Lond) 110: 267-278, 2006.

55. Krysiak R, Handzlik-Orlik G and Okopien B: The role of adipokines in connective tissue diseases. Eur J Nutr 51: 513-528, 2012.

56. Fantuzzi G: Adiponectin and inflammation: Consensus and controversy. J Allergy Clin Immunol 121: 326-330, 2008.

57. Nagaraju GP, Rajitha B, Aliya S, Kotipatruni RP, Madanraj AS, Hammond A, Park D, Chigurupati S, Alam A and Pattnaik S: The role of adiponectin in obesity-associated female-specific carcinogenesis. Cytokine Growth Factor Rev 31: 37-48, 2016.

58. Scotece M, Conde J, Gómez R, López V, Lago F, Gómez-Reino JJ and Gualillo O: Beyond fat mass: Exploring the role of adipokines in rheumatic diseases. ScientificWorldJournal 11: 1932-1947, 2011.

59. Beltowski J: Leptin and atherosclerosis. Atherosclerosis 189: 47-60, 2006.

60. Dini AA, Wang P and Ye DQ: Serum adiponectin levels in patients with systemic lupus erythematosus: A meta-analysis. J Clin Rheumatol 23: 361-367, 2017.

61. Liu D, Luo S and Li Z: Multifaceted roles of adiponectin in rheumatoid arthritis. Int Immunopharmacol 28: 1084-1090, 2015.

62. Wu XW, Ji HZ and Wang FY: Meta-analysis of ciprofloxacin in treatment of Crohn's disease. Biomed Rep 3: 70-74, 2015.

63. Zhao F, Wang Y, Liu L and Bian M: Erythropoietin for cancer-associated malignant anemia: A meta-analysis. Mol Clin Oncol 6: 925-930, 2017.

64. Yin M, Li G, Song $\mathrm{H}$ and Lin S: Identifying the association between interleukin- 6 and lichen planus: A meta-analysis. Biomed Rep 6: 571-575, 2017.

65. Li Y, Zeng X, He J, Gui Y, Zhao S, Chen H, Sun Q, Jia N and Yuan H: Circular RNA as a biomarker for cancer: A systematic meta-analysis. Oncol Lett 16: 4078-4084, 2018.

his work is licensed under a Creative Commons Attribution-NonCommercial-NoDerivatives 4.0 International (CC BY-NC-ND 4.0) License. 